\title{
Classification of COVID-19 patients from chest CT images using multi-objective differential evolution-based convolutional neural networks
}

\author{
Dilbag Singh $^{1} \cdot$ Vijay Kumar ${ }^{2} \cdot$ Vaishali $^{3} \cdot$ Manjit Kaur $^{3}$ \\ Received: 25 March 2020 / Accepted: 7 April 2020 / Published online: 27 April 2020 \\ (C) Springer-Verlag GmbH Germany, part of Springer Nature 2020
}

\begin{abstract}
Early classification of 2019 novel coronavirus disease (COVID-19) is essential for disease cure and control. Compared with reverse-transcription polymerase chain reaction (RT-PCR), chest computed tomography (CT) imaging may be a significantly more trustworthy, useful, and rapid technique to classify and evaluate COVID-19, specifically in the epidemic region. Almost all hospitals have CT imaging machines; therefore, the chest CT images can be utilized for early classification of COVID-19 patients. However, the chest CT-based COVID-19 classification involves a radiology expert and considerable time, which is valuable when COVID-19 infection is growing at rapid rate. Therefore, an automated analysis of chest CT images is desirable to save the medical professionals' precious time. In this paper, a convolutional neural networks (CNN) is used to classify the COVID-19-infected patients as infected (+ve) or not (-ve). Additionally, the initial parameters of CNN are tuned using multiobjective differential evolution (MODE). Extensive experiments are performed by considering the proposed and the competitive machine learning techniques on the chest CT images. Extensive analysis shows that the proposed model can classify the chest CT images at a good accuracy rate.
\end{abstract}

Keywords Coronavirus $\cdot$ Convolutional neural networks $\cdot$ Chest CT $\cdot$ Multi-objective $\cdot$ Differential evolution

\section{Introduction}

The novel coronavirus (nCoV) infection aroused in Wuhan, China, and has extensively spread all over the world since January 2020 [1]. World Health Organization (WHO) declared the outbreak of nCoV as a "Public Health Emergency of International Concern" on 30 January 2020 [2]. WHO named this disease as coronavirus disease (COVID-19) February 2020 [2]. To date (1 April 2020), there are 859,965 confirmed cases all around the world with 42,344 deaths and

Manjit Kaur

Manjit.kr@yahoo.com

1 Computer Science and Engineering Department, School of Computing and Information Technology, Manipal University Jaipur, Jaipur, India

2 Computer Science and Engineering Department, National Institute of Technology, Hamirpur, Himachal Pradesh, India

3 Computer and Communication Engineering Department, School of Computing and Information Technology, Manipal University Jaipur, Jaipur, India
178,364 recovered [3]. COVID-19 severely affected the USA (188,592 cases), Italy (105,972 cases), Spain (95,923 cases), China ( 81,554 cases), Germany ( 71,808 cases), France $(52,128$ cases $)$, and Iran $(44,605$ cases $)[3,4]$. The second largest population country in the world, i.e., India, is also affected from COVID-19 and 1718 confirmed cases with fifty-two deaths on 1 April 2020. The specific symptoms of COVID-19 are fever (98\%) and cough (76\%) in addition to other non-specific symptoms such as fatigue (44\%), headache $(8 \%)$, and dyspnea $(3 \%)[5,6,12]$.

There is a vital need to detect the disease at early stage and instantly quarantine the infected people due to unavailability of specific drugs for COVID-19. The Chinese Government reported that the diagnosis of COVID-19 is confirmed through real-time polymerase chain reaction (RT-PCR) [7]. However, RT-PCR suffers from high false-negative rates and timeconsuming [7-11]. The low sensitivity of RT-PCR is not acceptable in the current epidemic situation. In some cases, the infected people may not be recognized and get suitable treatment on time. The infected people may get spread the virus to healthy people due to communicable nature of $\mathrm{nCoV}$. It is observed from clinical reported of infected peoples that there 
is bilateral change in chest computed tomography (CT) images [8]. Therefore, chest $\mathrm{CT}$ has been used as alternative tool to detect the infection caused by $\mathrm{nCoV}$ due to high sensitivity [12]. The National Health Commission of China reported that chest $\mathrm{CT}$ can be utilized to detect the infection caused by $\mathrm{nCoV}$ [3]. A large amount of pathological information can be obtained from chest $\mathrm{CT}$. The radiologists are required to analyze the images of chest CT. Hence, there is a necessity to develop a deep learning-based prediction technique for analysis of chest $\mathrm{CT}$ without intervention of radiologist.

The main objective of this paper is to classify COVID-19infected patients from chest CT images. A novel deep learning model is designed by using multi-objective differential evolution (MODE) and convolutional neural networks (CNN) for classification of human beings based upon whether they are affected from COVID-19 or not. A multiobjective fitness function is designed to classify COVID19-infected patients by considering sensitivity and specificity. The hyperparameters of $\mathrm{CNN}$ are optimized by using the MODE algorithm. The proposed model is trained by considering the chest $\mathrm{CT}$ images of COVID-19 patients. The comparisons between the proposed MODE-based CNN with the competitive models such as convolutional neural networks (CNN), adaptive neuro-fuzzy inference systems (ANFIS), and artificial neural networks (ANN) are also drawn by considering the well-known classification metrics.

The remaining paper is summarized as follows: the "Literature review" section discusses the existing literature in the field of COVID-19; proposed classification model is discussed in the "Proposed model" section; performance analyses are discussed in the "Performance analysis" section; the "Conclusion" section concludes the paper.

\section{Literature review}

Recently, researchers have perceived the imaging patterns on chest CT for detecting the COVID-19 in chest CT [13-22]. Fang et al. [14] studied the sensitivity of RT-PCR and chest $\mathrm{CT}$ during the detection of COVID-19. They analyzed the travel history and symptoms of 2 patients and found that the sensitivity of chest CT for detection of COVID-19 is much higher than RT-PCR. Xie et al. [13] also reported that the 3\% of 167 patients had negative RT-PCR for COVID-19 detection. However, chest CT has better sensitivity of detection of COVID-19 over RT-PCR. Berheim et al. [23] studied 121 infected patients' chest CT from four different centers of China. The relationship between $\mathrm{CT}$ scan and symptom onset is established. They found that the severity of disease increased with time from onset of symptoms and designated the signs of disease. Recently, deep learning techniques have been widely used in detection of acute pneumonia in chest $\mathrm{CT}$ images [23-26].
Li et al. [24] developed a deep learning model named as COVNet to extract visual features from chest $\mathrm{CT}$ for detection of COVID-19. They used visual features to distinguish between community acquired pneumonia and other nonpneumonia lung diseases. However, COVNet is unable to categorize the severity of this disease. Gozes et al. [25] developed an artificial intelligence-based CT analysis tool for detecting and quantification of COVID-19. The system extracted slice of opacities in the lungs automatically. The developed system achieved $98.2 \%$ sensitivity and $92.2 \%$ specificity. The output of system provides quantitative opacity measure and $3 \mathrm{D}$ volume display for opacities. The system is robust against pixel spacing and slice thickness [25]. Shan et al. [26] developed a deep learning-based system named VB-net for automatic segmentation of all the lung and infection sites using chest $\mathrm{CT}$. Xu et al. [8] developed a prediction model to discriminate COVID-19 pneumonia and influenza-A viral pneumonia using deep learning techniques. The CNN model was used for prediction. The maximum accuracy obtained from prediction model was $86.7 \%$. Wang et al. [9] investigated the radiographic changes in $\mathrm{CT}$ images of infected patients. They developed a deep learning-based prediction model that utilizes the modified inception transfer learning technique. The features are extracted from CT images for prior diagnosis. The accuracy of $89.5 \%$ obtained from this method is better than Xu's model [8] and saved time for diagnosis. Narin et al. [11] proposed an automatic deep convolution neural networkbased transfer models for prediction of COVID-19 in chest Xray images. They used InceptionV3, Inception-ResNetV2, and ResNet50 models for better prediction. The ResNet50 pre-trained model produced accuracy of $98 \%$, which is higher than $[8,9]$. Sethy et al. [10] developed a deep learning model for detecting COVID-19 from X-ray images. They extracted deep features and transferred them to support vector machine for classification. The accuracy of $95.38 \%$ obtained from the proposed model, which is better than $[8,9]$.

From the extensive review, it has been found that the chest CT images can be used for early classification of COVID-19infected patients [27]. Therefore, in this paper, computational models are used to classify COVID-19 patients from chest CT images.

\section{Proposed model}

This section discusses the proposed multi-objective differential evolution (MODE)-based convolutional neural networks (CNN) for classification of COVID-19-infected patients from chest CT images. In this paper, initially, CNN, ANN, and ANFIS models are implemented to classify COVID-19infected patients from chest $\mathrm{CT}$ images. These models provide good performance for classification of COVID-19 patients. 
Although, CNN provides good results, but it suffers from hyperparameters tuning issue.

\section{Convolutional neural networks}

Convolutional neural networks (CNN) [28] is a powerful tool which is extensively utilized for image classification. The hierarchical structure and efficient feature extraction characteristics from an image make $\mathrm{CNN}$ a dynamic model for image classification. Initially, the layers are organized in three dimensions: width, height, and depth. The neurons in given layer do not attach to entire set of neurons in the later layer, but only to a limited neurons of it. Finally, an output is diminished to a single vector of probability scores, coordinated alongside the depth dimension. The training and testing frameworks of the deep convolutional models for COVID-19 classification is shown in Fig. 1. It demonstrates that the $\mathrm{CNN}$ classifier utilizes various layers for model building and testing purpose.

For classification of COVID-19-infected patients, features of chest CT images are used to accurately classify the patients whether they belong to infected class or not. The process of chest CT image based COVID-19 from disease classification also involves repeated classification calculations and computations. To classify COVID-19-infected patients by using the CNN model, the following steps are used:

A. Feature extraction

In this step, $\mathrm{CNN}$ implements several convolutions and pooling operations to evaluate and monitor potential features [29]. Figure 2 shows how kernel/filter can extract potential features by using the stride.

Max pooling layer is then utilized to minimize the spatial size of the convolved features. It has an ability to overcome the overfitting issue. It considers maximum of the region from feature map obtained from convolution operator [30]. Figure 3 shows an example of a max pooling layer's with a kernel size of 2 and stride of 1 .

Rectified linear unit (ReLU) activation function is used to learn complex functional mappings between the inputs and response variables $[31,32]$. It is a linear function that produces the input directly if it is positive; otherwise, it will output zero (see Fig. 4).

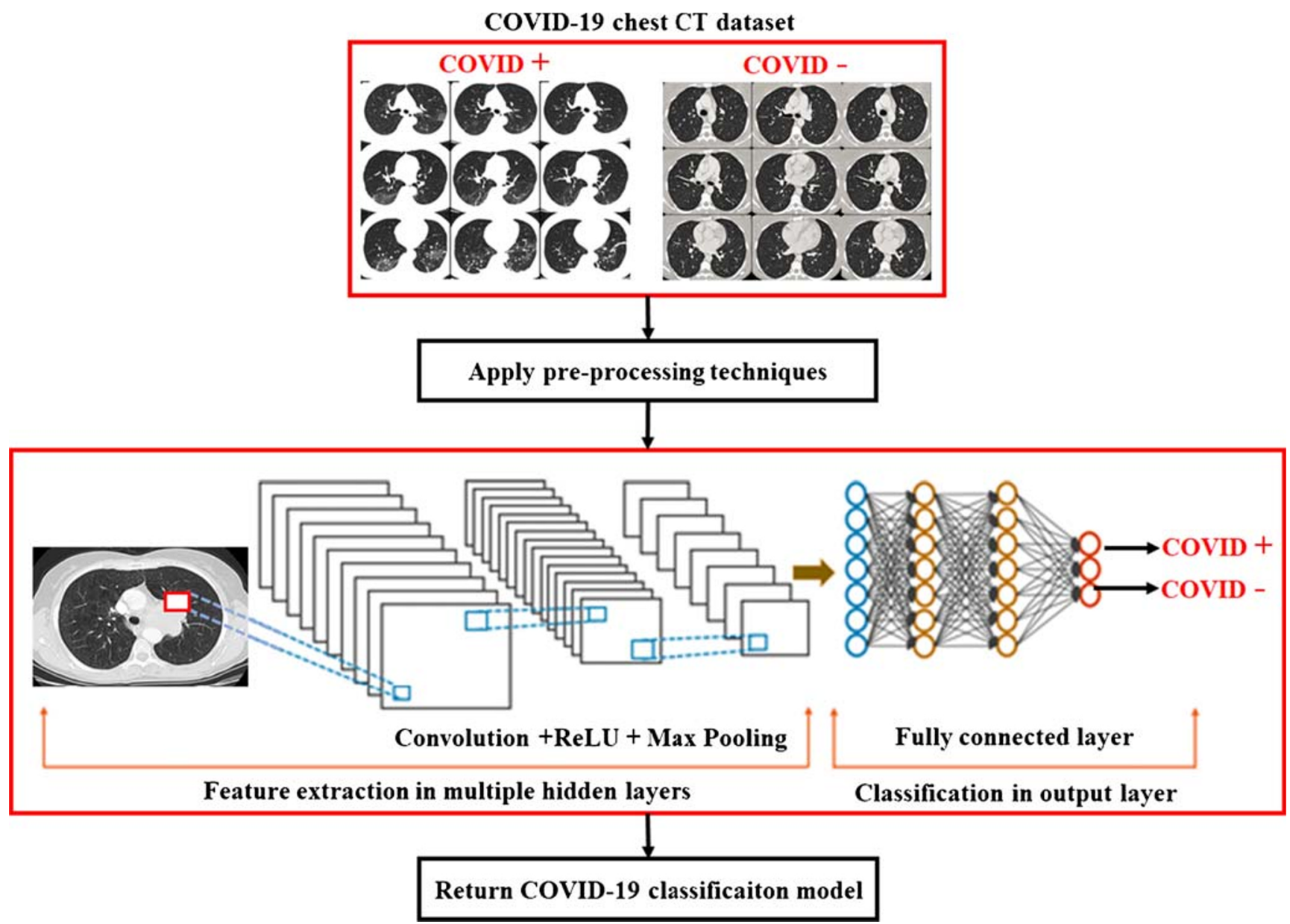

Fig. 1 Block diagram of the training process of the CNN-based COVID-19 classification model 
Fig. 2 Convolution operator with kernel size 3 and stride 2

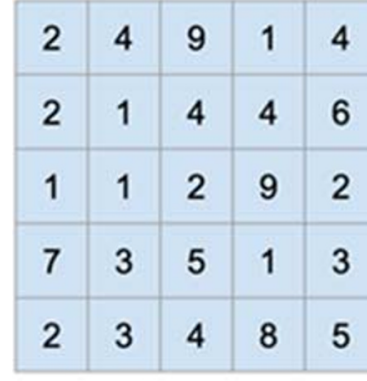

Image

\begin{tabular}{|c|c|c|}
\hline 1 & 2 & 3 \\
\hline-4 & 7 & 4 \\
\hline 2 & -5 & 1 \\
\hline
\end{tabular}

Filter I

Kernel

\section{B. Classification}

In this step, fully connected layers act as a classifier. It utilizes extracted features and evaluates probability for object in the image [33]. Usually, activation function and dropout layer are utilized to establish non-linearity and minimize overfitting, respectively [34]. Figure 5 shows the fully connected layer used for the classification process.

\section{Multi-objective fitness function}

From literature review, it has been found that CNN suffers from hyperparameter tuning issues. These hyperparameters are kernel size, kernel type, stride, padding, hidden layer, activation functions, learning rate, momentum, number of epochs, and batch size. Therefore, the tuning of these parameters is desirable. In this paper, a multi-objective fitness function is designed as:

$f(t)=S_{n}+S_{p}$

Here, $S_{n}$ and $S_{p}$ define the sensitivity and specificity parameters, respectively.

Sensitivity, i.e., true positive rate, computes the ratio of actual positives that are correctly classified. Confusion matrix is utilized to evaluate the sensitivity $\left(S_{n}\right)$ and it is mathematically evaluated as [35]:

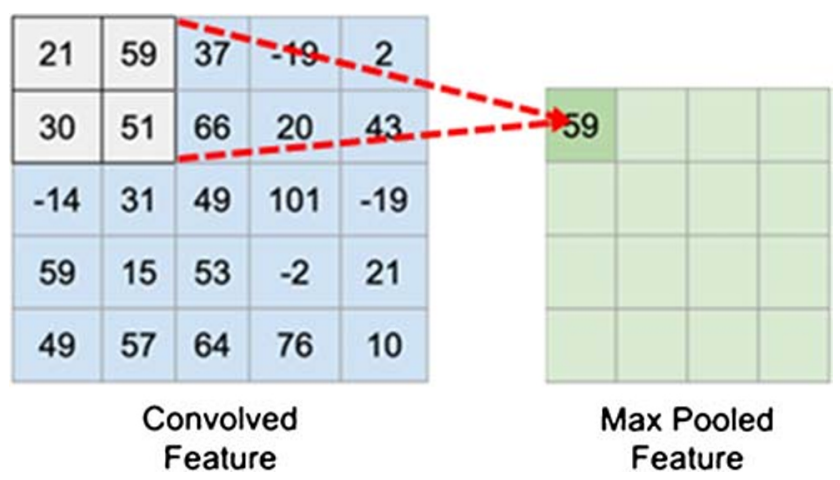

Fig. 3 Max pooling with one pooled feature
$S_{n}=\frac{T_{p}}{T_{p}+F_{n}}$

Here, $T_{p}$ and $F_{n}$ define true positive and false-negative values, respectively. $S_{n}$ lies within $[0,100] . S_{n}$ approaching towards 100 is desirable [36].

Specificity $\left(S_{p}\right)$ computes the proportion of actual negatives that are correctly identified and it can be estimated as [37]:

$S_{p}=\frac{T_{n}}{T_{n}+F_{p}}$

Here, $T_{n}$ and $F_{p}$, define true negative rate and false-positive values, respectively. $S_{n}$ lies within $[0,100] . S_{p}$ approaching towards 100 is desirable [38].

\section{Multi-objective differential evolution}

The idea of differential evolution (DE) was coined by Storn and Price [39] in 1995. DE has got its inspiration from Darwin's theory of evolution and natural selection. Over the time, many DE variants have been introduced [40-42]. DE algorithm has proven its potency in various domains [41,

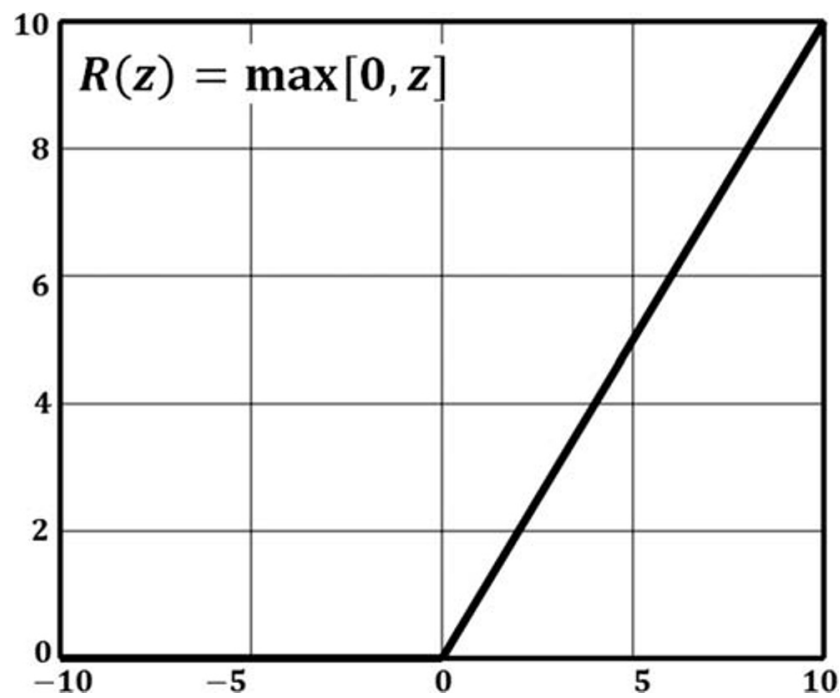

Fig. 4 Rectified linear unit (ReLU) activation function 
Table 1 Initial parameters of the MODE algorithm

\begin{tabular}{ll}
\hline Parameter & Value/range \\
\hline [Lower bound and upper bound] & {$[0,1]$} \\
Population size & 40 \\
Population type & Double vector \\
Crossover rate & 0.5 \\
Selection & Tournament \\
Termination criteria & Generations: 200 \\
Scaling factor & 0.1 \\
\hline
\end{tabular}

43-45]. In DE algorithm, the population of candidate solution evolves iteratively using mutation, crossover, and selection operation to find out the best available solution [42]. This evolution from one generation to another ensures that the individual has better qualities remains part of the population and weak individuals are removed with each iteration [43]. The quality of each individual is calculated with the help of a predefined fitness/objective function [44].

For optimizing a problem with $\mathrm{DE}$, the population $(N P)$ of candidate solutions (having predefined upper and lower bound) is initialized randomly. Each individual of the population represented as $x_{a}$ consists of $D$ variables. Mutation, crossover, and selection operations for this population are carried out as follows $[39,45]$ :

\section{A. Mutation operation}

In this phase, a mutant/donor vector $\left(V_{a}\right)$ is created for each target vector $\left(X_{a}\right)$ in the population as:

$V_{a, g}=x_{r 1, g}+F\left(x_{r 2, g}-x_{r 3, g}\right)$

Here, $g$ represents generation. $F$ is scaling factor/ mutation parameter. $F$ amplifies the difference vector and lies within $[0$, 1]. $r_{1}, r_{2}$, and $r_{3}$ are randomly chosen numbers from $[1, N P]$ such that $r_{1} \neq r_{2} \neq r_{3} \neq a$.

The best vector of the population can also be used to produce mutant vector [46] as:

$V_{a, g}=x_{\text {best }, g}+F\left(x_{r 1, g}-x_{r 2, g}\right)$

\section{B. Crossover operation}

The crossover could be binomial or exponential. In both, the trial vector, denoted by $U$, is created with the combination of mutant vectors and target vectors according to predefined conditions. Binomial crossover is performed as:

$U_{b, a, g}=\left\{\begin{array}{cr}V_{b, a, g} & \text { if } \operatorname{rand}_{a, b}[0,1] \leq C_{R} \quad \text { or } b=b_{\text {rand }} \\ X_{b, a, g} & \text { Otherwise }\end{array}\right.$

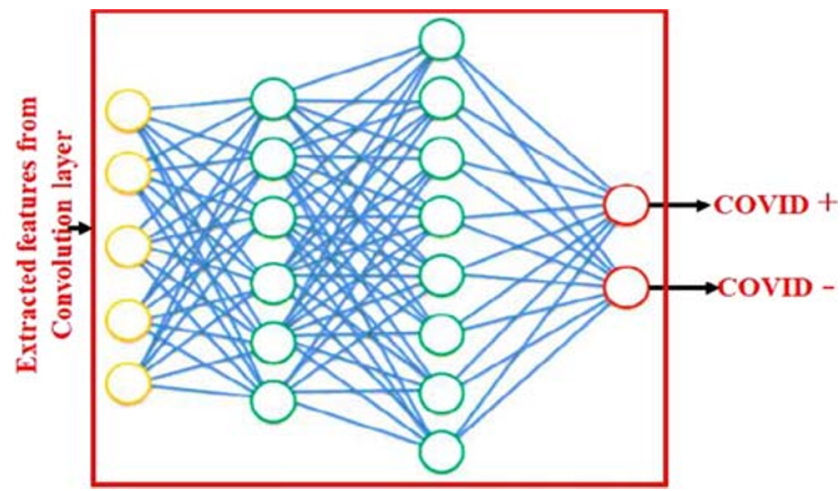

Fig. 5 Fully connected layer

Here, $C_{R}$ is crossover rate in the range $[0,1] . a=1,2, \ldots$. $N P$ and $b=1,2, \ldots, D . b_{\text {rand }}$ are a randomly selected variable of the mutant vector which ensure that the trial vector is not simply a replica of target vector. In exponential crossover also, a random variable is chosen initially, and $e$ consecutive components are chosen circularly from donor/mutant vector. The probability with which, $i$ th element is replaced in $\{1,2, \ldots$, $e\}$, decreases exponentially as $i$ increases. The pseudo-code for exponential crossover is as follows:

Algorithm 1 Exponential crossover

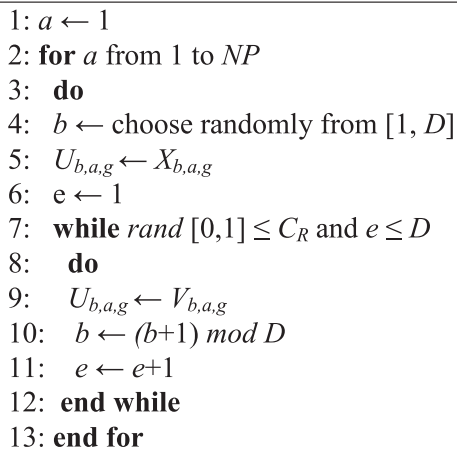

\section{Selection operation}

In this phase, the decision vector will move to the next generation. This greedy selection depends upon the fitness value of the decision vector. The vector with better fitness participates further in evolution of the next generation $(g+1)$. The selection operation is carried out as:

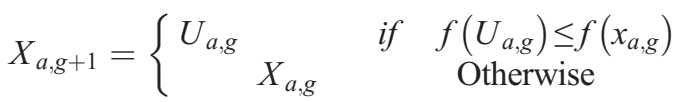

The above-mentioned operations are performed on the population until the termination criteria is satisfied. The termination condition for $\mathrm{DE}$ can be determined by the number of iterations or the maximum number of function evaluations. 
Fig. 6 COVID-19 patient chest CT images (adapted from [20]) (a-d)

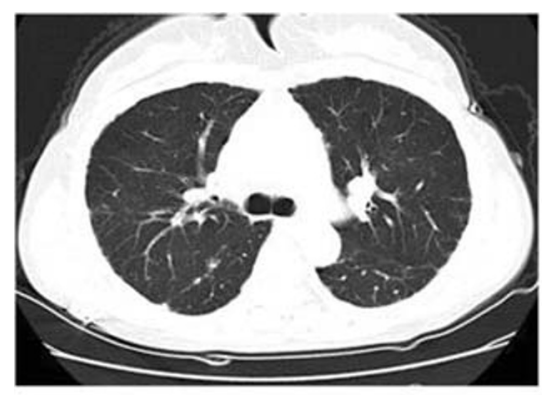

a

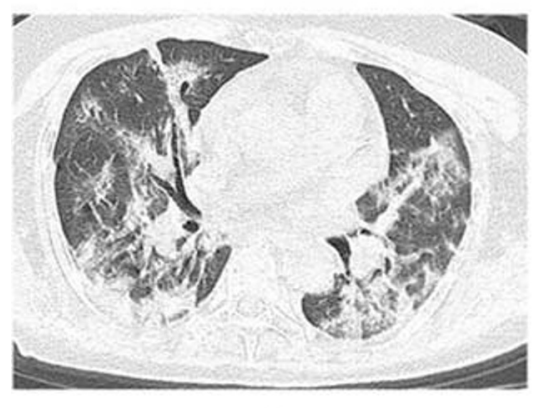

C

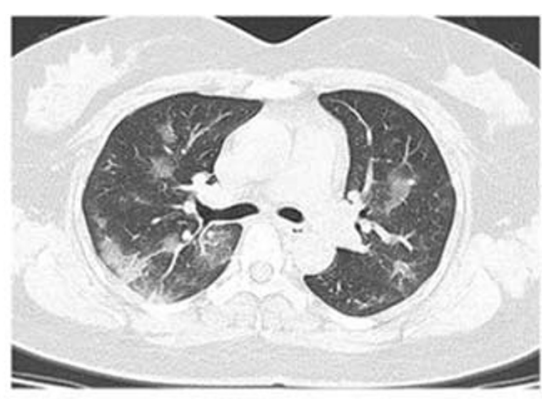

b

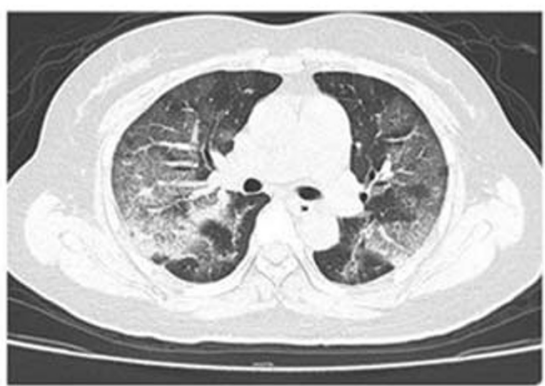

d

\section{Performance analysis}

The proposed COVID-19 classification model is implemented using MATLAB 2019a software with deep learning toolbox.

a

\begin{tabular}{|c|c|c|}
\cline { 2 - 3 } \multicolumn{1}{c|}{} & $\begin{array}{c}\text { Actual COVID-19 } \\
=\text { Yes (+) }\end{array}$ & $\begin{array}{c}\text { Actual COVID-19 } \\
=\text { Yes (-) }\end{array}$ \\
\hline $\begin{array}{c}\text { Predicted } \\
\text { COVID-19 } \\
=\text { Yes (+) }\end{array}$ & $\begin{array}{c}\text { True Positive } \\
\text { TP= } 60 \pm 6\end{array}$ & $\begin{array}{c}\text { False Positive } \\
\text { FP=8 } \pm 3\end{array}$ \\
\hline $\begin{array}{c}\text { Predicted } \\
\text { COVID-19 } \\
=\text { Yes (-) }\end{array}$ & $\begin{array}{c}\text { False Negative } \\
\text { FN =9 } \pm 4\end{array}$ & $\begin{array}{c}\text { True Negative } \\
\text { TN=56 } \pm 6\end{array}$ \\
\hline
\end{tabular}

C

\begin{tabular}{|c|c|c|}
\cline { 2 - 3 } \multicolumn{1}{c|}{} & $\begin{array}{c}\text { Actual COVID-19 } \\
=\text { Yes (+) }\end{array}$ & $\begin{array}{c}\text { Actual COVID-19 } \\
\text { = Yes (-) }\end{array}$ \\
\hline $\begin{array}{c}\text { Predicted } \\
\text { COVID-19 } \\
=\text { Yes (+) }\end{array}$ & $\begin{array}{c}\text { True Positive } \\
\text { TP= 58 } \pm 5\end{array}$ & $\begin{array}{c}\text { False Positive } \\
\text { FP=9 } \pm 3\end{array}$ \\
\hline $\begin{array}{c}\text { Predicted } \\
\text { COVID-19 } \\
=\text { Yes (-) }\end{array}$ & $\begin{array}{c}\text { False Negative } \\
\text { FN =10 } \pm 5\end{array}$ & $\begin{array}{c}\text { True Negative } \\
\text { TN= 55 } \pm 6\end{array}$ \\
\hline
\end{tabular}

Intel Core i7 with 16-GB RAM and 4-GB graphics card is used. 20.80 cross-validation is used to prevent the overfitting problem. Various variations of training and testing dataset ratio such as 20,80\%, 30:70\%, 40:60\%, 50:50\%, 60:40\%,

\begin{tabular}{|c|c|c|}
\cline { 2 - 3 } \multicolumn{1}{c|}{} & $\begin{array}{c}\text { Actual COVID-19 } \\
=\text { Yes (+) }\end{array}$ & $\begin{array}{c}\text { Actual COVID-19 } \\
=\text { Yes (-) }\end{array}$ \\
\hline $\begin{array}{c}\text { Predicted } \\
\text { COVID-19 } \\
=\text { Yes (+) }\end{array}$ & $\begin{array}{c}\text { True Positive } \\
\text { TP=59 } \pm 4\end{array}$ & $\begin{array}{c}\text { False Positive } \\
\text { FP=10 } \pm 3\end{array}$ \\
\hline $\begin{array}{c}\text { Predicted } \\
\text { COVID-19 } \\
=\text { Yes (-) }\end{array}$ & $\begin{array}{c}\text { False Negative } \\
\text { FN =9 } \pm 6\end{array}$ & $\begin{array}{c}\text { True Negative } \\
\text { TN= 54 } \pm 7\end{array}$ \\
\hline
\end{tabular}

b

\begin{tabular}{|c|c|c|}
\cline { 2 - 3 } \multicolumn{1}{c|}{} & $\begin{array}{c}\text { Actual COVID-19 } \\
=\text { Yes (+) }\end{array}$ & $\begin{array}{c}\text { Actual COVID-19 } \\
=\text { Yes (-) }\end{array}$ \\
\hline $\begin{array}{c}\text { Predicted } \\
\text { COVID-19 } \\
=\text { Yes }(+)\end{array}$ & $\begin{array}{c}\text { True Positive } \\
\text { TP= 62 } \pm 4\end{array}$ & $\begin{array}{c}\text { False Positive } \\
\text { FP }=7 \pm 3\end{array}$ \\
\hline $\begin{array}{c}\text { Predicted } \\
\text { COVID-19 } \\
=\text { Yes }(-)\end{array}$ & $\begin{array}{c}\text { False Negative } \\
\text { FN }=6 \pm 5\end{array}$ & $\begin{array}{c}\text { True Negative } \\
\text { TN= 58 } \pm 6\end{array}$ \\
\hline \multicolumn{3}{|c|}{ d } \\
\hline
\end{tabular}

Fig. 7 Confusion matrix (i.e., error matrix) analysis of a ANN, b ANFIS, $\mathbf{c}$ CNN, and the proposed MODE-based CNN model 
Fig. 8 Receiver operating characteristic (ROC) analysis

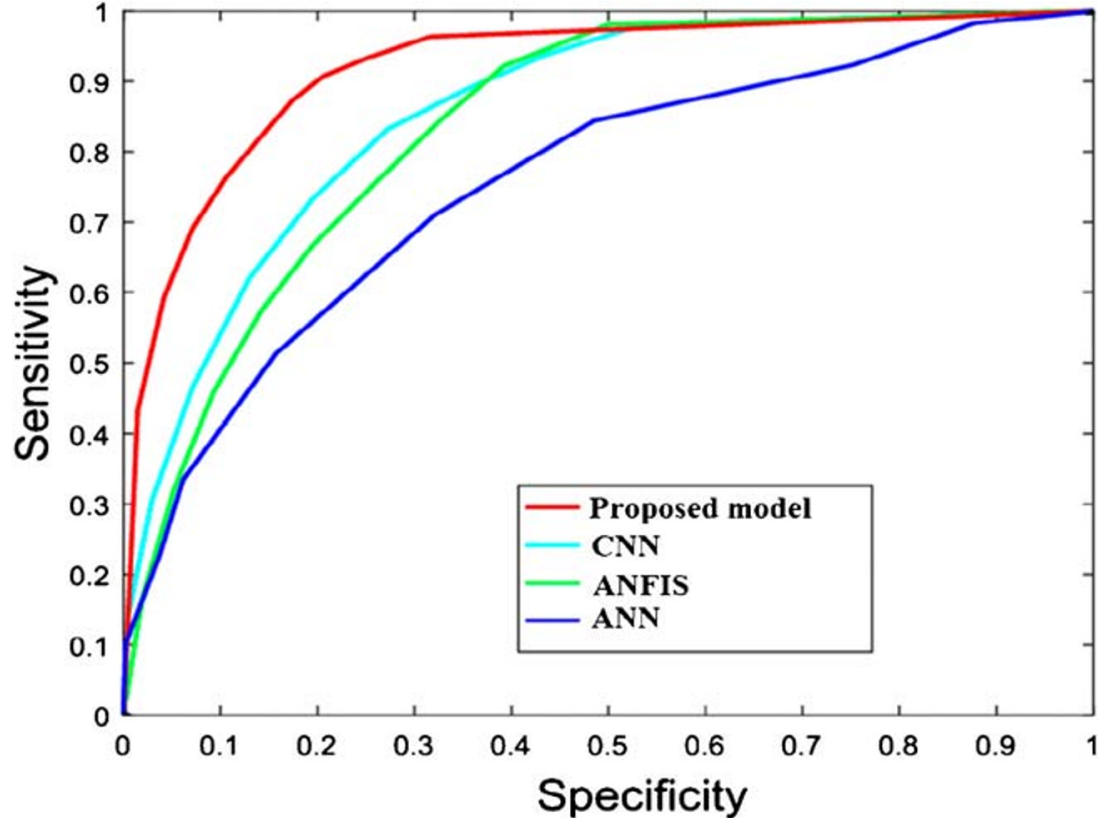

70:30\%, 80:20\%, and 90:10\%, respectively are considered for experimental purpose. The proposed model is compared with CNN, ANFIS, and ANN models. Table 1 shows the initial parameters of MODE to tune CNN.

\section{COVID-19 chest CT images dataset}

The 2019 novel coronavirus (COVID-19) shows a number of unique characteristics. COVID-19's infection can be classified by considering the polymerase chain reaction. It is found that the COVID-19-infected patients show some pattern on chest CT images which is not easily detectable by human eye.
COVID-19 patients present abnormalities in chest CT images with most having bilateral involvement. Bilateral multiple lobular and subsegmental areas of consolidation constitute the typical findings in chest CT images of intensive care unit (ICU) patients on admission. In comparison, non-ICU patients show bilateral ground-glass opacity and subsegmental areas of consolidation in their chest CT images. In these patients, later chest CT images display bilateral ground-glass opacity with resolved consolidation [27]. Therefore, in this paper, chest CT images dataset is used to classify the COVID-19.

Figure 6 shows different chest CT images COVID-19infected patients. Figure 6 a shows an axial CT image of mild

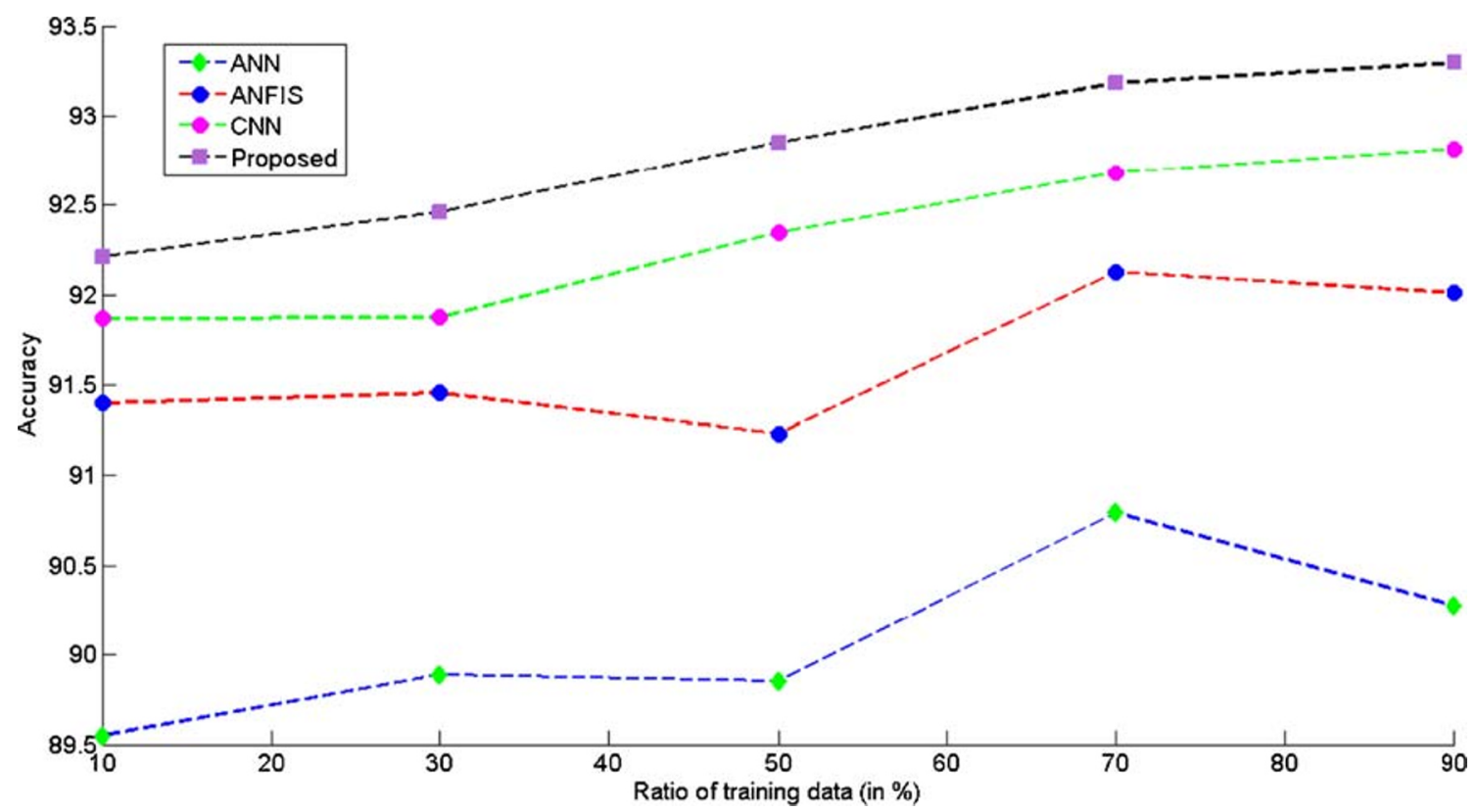

Fig. 9 Accuracy analysis of the proposed and competitive COVID-19 classification models 


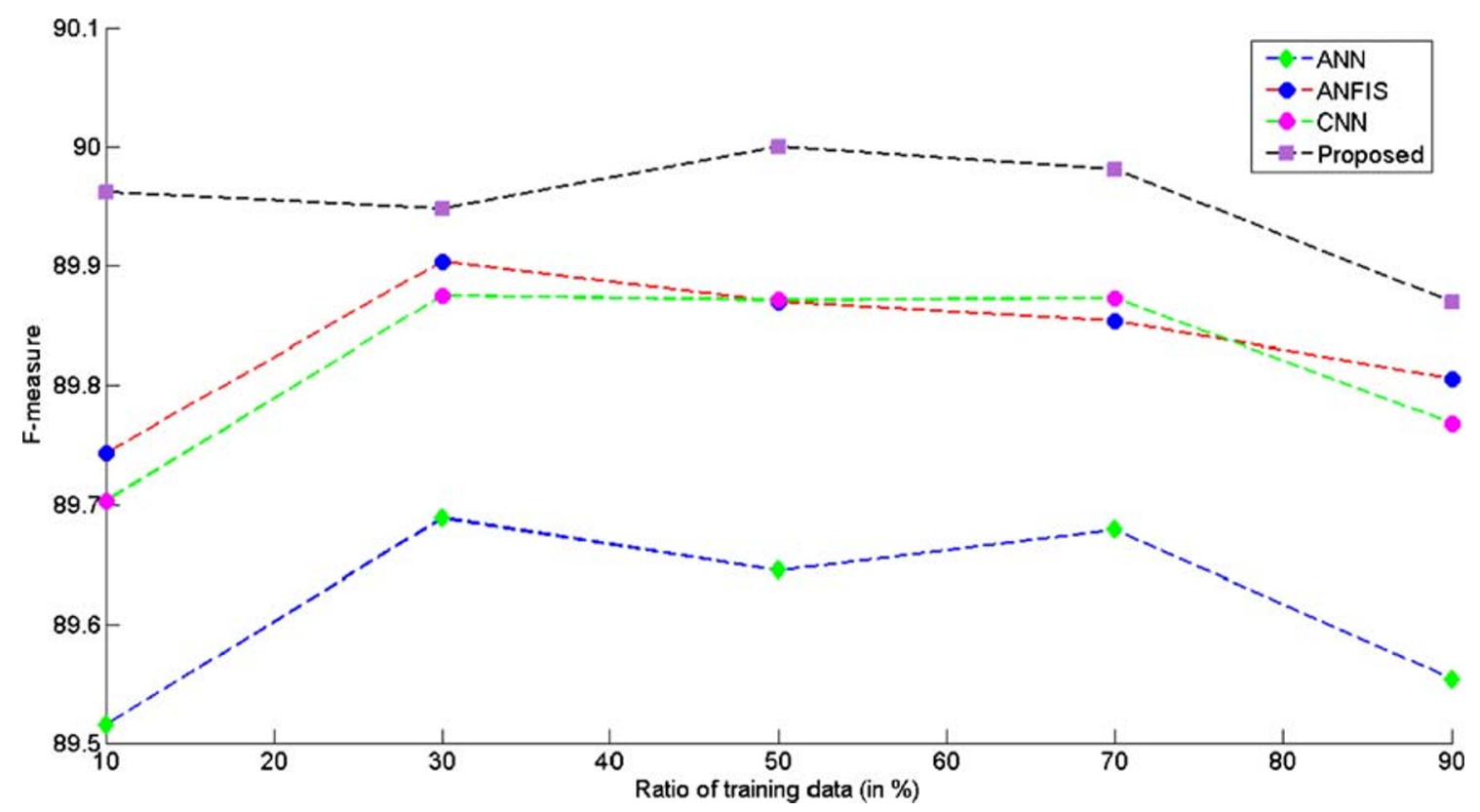

Fig. 10 F-measure analysis of the proposed and competitive COVID-19 classification models

type patient (2 days from symptom onset to CT scan). It demonstrates thickening of the lung texture. Figure $6 \mathrm{~b}$ shows an axial CT image of common type patient (6 days from symptom onset to CT scan). It illustrates multiple ground-glass opacities in both lungs. Figures $6 \mathrm{c}$ shows an axial CT image of severe type patient. It demonstrates extensive ground-glass opacities and pulmonary consolidation, enlargement of bronchi, and vessels. Figure $6 \mathrm{~d}$ demonstrates an axial CT image of critical type patient (9 days from symptom onset to CT scan). It illustrates extensive ground-glass opacities in several lobes, formatting "white lung."

\section{Quantitative analysis}

Figure 7 shows the confusion matrix analysis of the proposed and the competitive models for COVID-19 disease classification. It is found that the proposed MODE-based CNN model outperforms the competitive models as it has better, and consistent true positive and true negative values as compared with

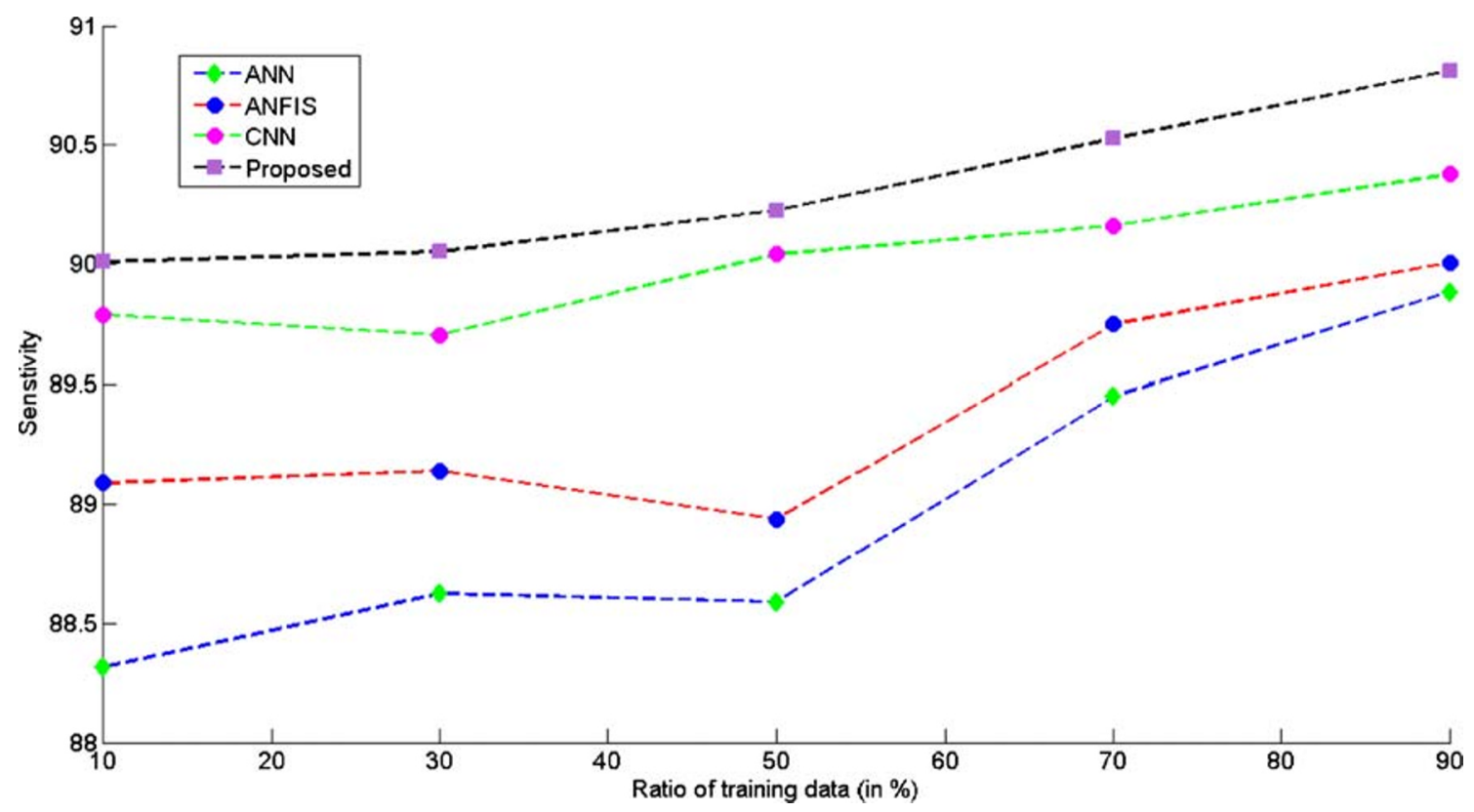

Fig. 11 Sensitivity analysis of the proposed and competitive COVID-19 classification models 


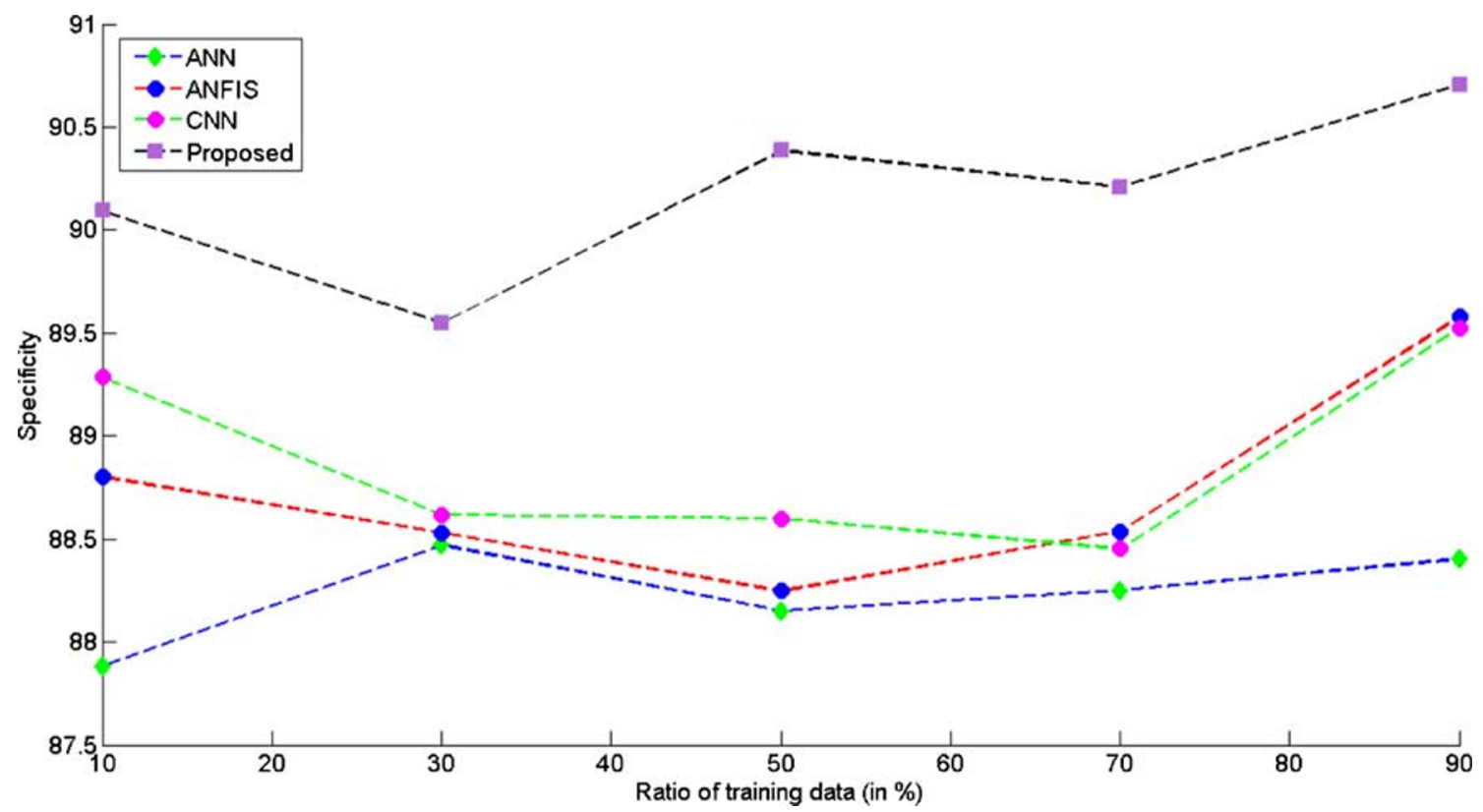

Fig. 12 Specificity analysis of the proposed and competitive COVID-19 classification models

other models. Also, it shows that the proposed model has lesser false-negative and false-positive values. Therefore, the proposed model can efficiently classify the COVID-19 patients.

Receiver operating characteristic (ROC) is a performance measurement curve for classification problem by considering number of threshold values. It is defined as a probability curve that defines the degree separability between two classes such COVID-19 (+) and COVID-19 (-). It evaluates the performance of classification models for distinguishing between COVID-19 (+) and COVID-19 (-). Higher the ROC, better the classification model is at classifying COVID-19 (+)s as
COVID-19 (+)s and vice versa. Figure 8 shows the obtained ROC of the proposed and competitive classification models. It clearly shows that the proposed model achieves good results as compared with the competitive models.

Accuracy is computed by dividing the accurately classified classes by total number of classes. It is a primary measure to compute the performance of classification problems. Figure 9 shows the accuracy analysis between the proposed and competitive classification models. It clearly shows that the proposed model achieves significantly more accuracy as compared with the competitive classification models. The

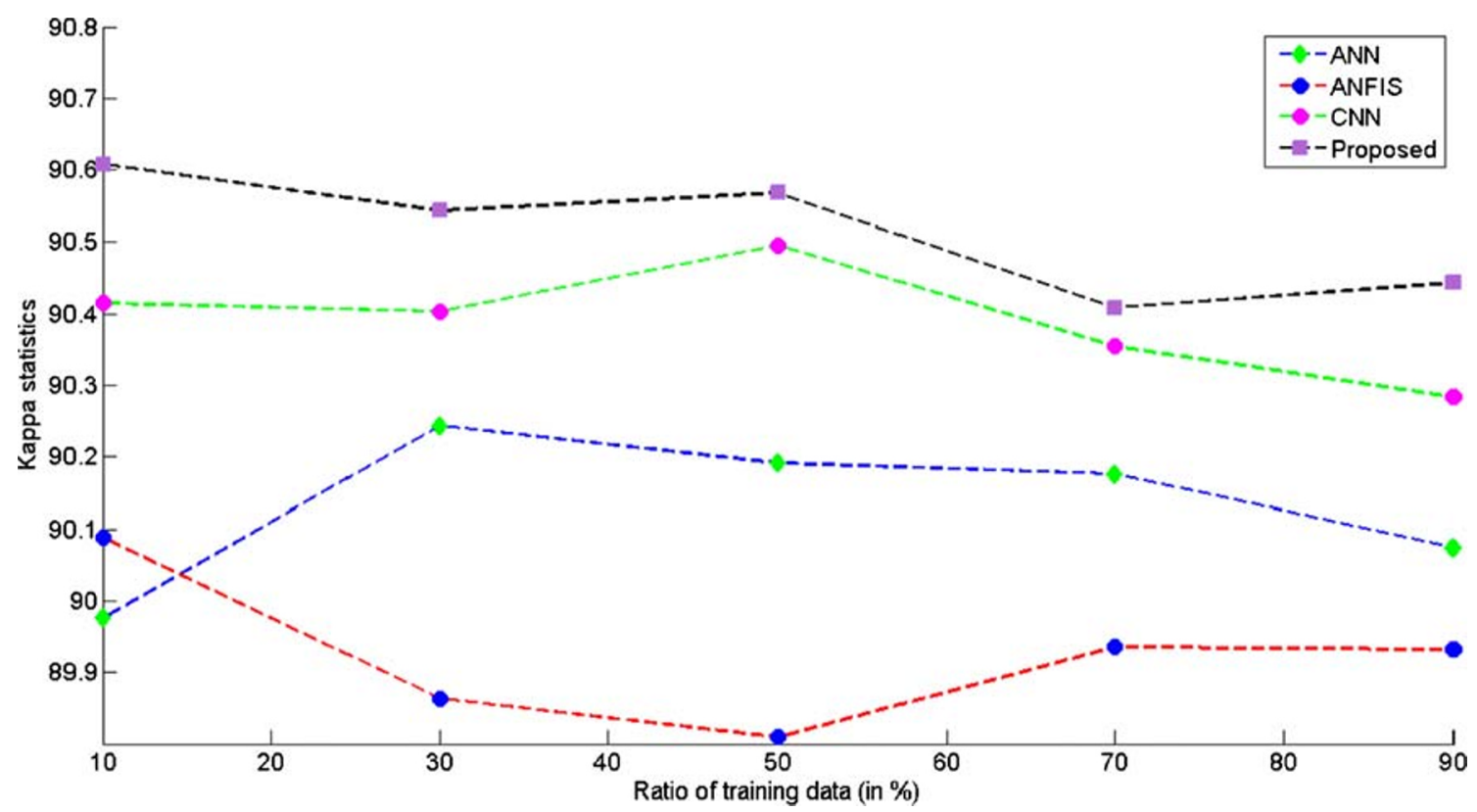

Fig. 13 Kappa statistics analysis of the proposed and competitive COVID-19 classification models 
proposed model outperforms competitive models by $1.9789 \%$.

F-measure is a well-known measure which can provide significant details of classification problems especially when data contain imbalanced classes. It calculates weighted harmonic mean of the recall and precision. Figure 10 demonstrates the F-measure analysis between the proposed and competitive classification models. It reveals that the proposed model achieves significantly more F-measure as compared with the competitive classification models. The proposed model outperforms competitive models by $2.0928 \%$.

Sensitivity computes the performance of the COVID-19 $(+)$ cases only. Thus, this test identifies very patient who is actually infected from COVID-19 disease. Figure 11 shows the sensitivity analysis between the proposed and competitive classification models. It reveals that the proposed model achieves significantly more sensitivity as compared with the competitive classification models. The proposed model outperforms competitive models by $1.8262 \%$.

Specificity evaluates the performance of the COVID-19 (-) cases only. Thus, this test identifies every patient who is not infected from COVID-19 disease. Figure 12 depicts the specificity analysis between the proposed and competitive classification models. It reveals that the proposed model achieves significantly more specificity as compared with the competitive classification models. The proposed model outperforms competitive models by $1.6827 \%$.

Kappa statistics is a performance metric used to evaluate the linear inter-rater reliability. It is also known as a reliable measure. It considers expected value by subtracting it from the classification success. Figure 13 depicts the Kappa statistics analysis between the proposed and competitive classification models. It reveals that the proposed model achieves significantly more Kappa statistics values as compared with the competitive classification models. The proposed model outperforms competitive models by $1.9276 \%$.

\section{Conclusion}

In this paper, a COVID-19 disease classification model is proposed to classify the infected patients from chest $\mathrm{CT}$ images. Initially, the chest CT dataset of COVID-19-infected patients is decomposed into training and testing groups. The training dataset is utilized for building the COVID-19 disease classification model. The proposed MODE-based CNN and competitive classification models are applied on the training data. To prevent the overfitting, 20 -fold cross-validation is also utilized. Finally, the comparisons are drawn between the competitive and proposed classification models by considering different fractions of training and testing dataset. Extensive experimental results reveal that the proposed model outperforms competitive models, i.e., ANN, ANFIS, and
CNN models in terms of accuracy, F-measure, sensitivity, specificity, and Kappa statistics by $1.9789 \%, 2.0928 \%$, $1.8262 \%, 1.6827 \%$, and $1.9276 \%$, respectively. Therefore, the proposed model is useful for real-time COVID-19 disease classification from chest $\mathrm{CT}$ images.

\section{Compliance with ethical standards}

Conflict of interest The authors declare that they have no conflict of interest.

Ethical approval This research work does not involve chemicals, procedures, or equipment that have any unusual hazards inherent in their use.

Informed consent Not required.

\section{References}

1. World Health Organization, Novel Coronavirus(2019-nCoV) Situation Report-11. 2020. https://www.who.int/docs/defaultsource/coronaviruse/situationreports/20200131-sitrep-11-ncov. pdf?sfvrsn=de7c0f7_4. Accessed 24 March 2020

2. World Health Organization, Novel Coronavirus(2019-nCoV) Situation Report-30. https://www.who.int/docs/default-source/ coronaviruse/situationreports/20200219-sitrep-30-covid-19.pdf? sfvrsn=6e50645_2. Accessed 24 March 2020

3. Worldometer Coronavirus. https://www.worldmeters.info/ coronavirus/countries-where-coronavirus-has-spread/. Accessed 01 Apr 2020

4. WHO-China joint mission on coronavirus disease 2019 report, 2020

5. Zhang Y (2020) The epidemiological characteristics of an outbreak of 2019 novel coronavirus (COVID-19)-China CCDC. Zhonghua liu xing bing xue za zhi=Zhonghua liuxingbingxue zazhi 41(2):145

6. Xie Z (2020) Pay attention to SARS-CoV-2 infection in children. Pediatr Invest 4(1):1-4

7. Ali T et al (2020) Correlation of chest CT and RT-PCR testing in coronavirus disease 2019 (COVID-19) in China: a report of 1014 cases. Radiology. https://doi.org/10.1148/radiol.2020200642

8. Xu X, Jiang X, Ma C, Du P, Li X, Lv S, Yu L, Chen Y, Su J, Lang G, Li Y, Zhao H, Xu K, Ruan L, Wu W (2020) Deep learning system to screen coronavirus disease 2019 pneumonia. arXiv preprintarXiv: 2002.09334, 1-29

9. Wang S, Kang B, Ma J, Zeng X, Xiao M, Guo J,Cai M, Yang J, Li Y, Meng X, Xu B (2020) A deep learning algorithm using CT images to screen for corona virus disease (COVID-19). medRxiv preprint. https://doi.org/10.1101/2020.02.14.20023028, 1-26

10. Sethy PK, Behera SK Detection of coronavirus disease (COVID19) based on deep features. Preprints 2020, 2020030300. https:// doi.org/10.20944/preprints202003.0300.v1

11. Narin A, Kaya C, Pamuk Z (2020) Automatic detection of coronavirus disease (COVID-19) using X-ray images and deep convolutional neural network. arXiv preprint arXiv:2003.10849

12. Wang D, Hu B, Hu C et al (2020) Clinical characteristics of 138 hospitalized patients with 2019 novel coronavirus-infected pneumonia in Wuhan, China. Jama 323(11):1061-1069

13. Xie X, Zhong Z, Zhao W, Zheng C, Wang F, Liu J (2020) Chest CT for typical 2019-nCoV pneumonia: relationship to negative RTPCR testing. Radiology. https://doi.org/10.1148/radiol.2020200343 
14. Fang Y, Zhang H, Xu Y, Xie J, Pang P, Ji W (2020) CT manifestations of two cases of 2019 novel coronavirus (2019-nCoV) pneumonia. Radiology 295(1):208-209

15. Song F, Shi N, Shan F et al (2020) Emerging coronavirus 2019nCoV pneumonia. Radiology 295(1)210-217

16. Ng M, Lee E, Yang J et al (2020) Imaging profile of the COVID-19 infection: radiologic findings and literature review. Radiol Cardiothorac Imaging 2(1):e200034

17. Kong W, Agarwal P (2020) Chest imaging appearance of COVID19 infection. Radiol Cardiothorac Imaging. https://doi.org/10.1148/ ryct.2020200028

18. Kay F, Abbara S (2020) The many faces of COVID-19: spectrum of imaging manifestations. Radiol Cardiothorac Imaging. https://doi. org/10.1016/B978-0-12-814551-7.00038-6

19. Venugopal VK, Mahajan V, Rajan S, Agarwal VK, Rajan R, Syed S, Mahajan H (2020) A systematic meta-analysis of CT features of COVID-19: lessons from radiology. medRxiv. https://doi.org/10. 1101/2020.04.04.20052241

20. Li X, Zeng X, Liu B, Yu Y (2020) COVID-19 infection presenting with CT halo sign. Radiol Cardiothorac Imaging. https://doi.org/10. 1148/ryct.2020200026

21. Chung M, Bernheim A, Mei X et al (2020) CT imaging features of 2019 novel coronavirus (2019-nCoV). Radiology 295(1):202-207

22. Yijiu X China's Hubei reports jump in new cases of COVID-19 after diagnosis criteria revision. National Health Commission of the People's Republic of China website. www.en.nhc.gov/cn/2020-02/ 13/c 76515.htm. Accessed 24 March 2020

23. Bernheim A, Mei X, Huang M et al (2020) Chest CT findings in coronavirus disease-19 (COVID-19): relationship to duration of infection https://doi.org/10.1148/radiol.2020200463

24. Li L et al (2020) Artificial intelligence distinguishes COVID-19 from community acquired pneumonia on chest CT. Radiology. https://doi.org/10.1148/radiol.2020200905

25. Gozes O et al (2020) Rapid AI development cycle for the coronavirus (COVID-19) pandemic: initial results for automated Detection \& patient monitoring using deep learning CT image analysis. arXiv preprint arXiv:2003.05037

26. Shan F, Gao Y, Wang J, Shi W, Shi N, Han M, Xue Z, Shi Y (2020) Lung infection quantification of COVID-19 in CT images with deep learning. arXiv preprint arXiv:2003.04655, 1-19, 2020

27. Liu K-C, Xu P, Lv W-F, Qiu X-H, Yao J-L, Jin-Feng G (2020) CT manifestations of coronavirus disease-2019: a retrospective analysis of 73 cases by disease severity. Eur J Radiol 108941. https://doi.org/ 10.1016/j.ejrad.2020.108941

28. Moeskops P, Viergever MA, Mendrik AM, De Vries LS, Benders MJNL, Išgum I (2016) Automatic segmentation of MR brain images with a convolutional neural network. IEEE Trans Med Imaging 35(5):1252-1261

29. Gu J, Wang Z, Kuen J, Ma L, Shahroudy A, Shuai B, Liu T et al (2018) Recent advances in convolutional neural networks. Pattern Recogn 77:354-377

30. Matsugu M, Mori K, Mitari Y, Kaneda Y (2003) Subject independent facial expression recognition with robust face detection using a convolutional neural network. Neural Netw 16(5-6):555-559
31. Krizhevsky A, Sutskever I, Hinton GE (2012) Imagenet classification with deep convolutional neural networks. In: Advances in neural information processing systems, pp 1097-1105

32. Jin KH, McCann MT, Froustey E, Unser M (2017) Deep convolutional neural network for inverse problems in imaging. IEEE Trans Image Process 26(9):4509-4522

33. Zbontar J, LeCun Y (2016) Stereo matching by training a convolutional neural network to compare image patches. J Mach Learn Res 17(1):2287-2318

34. Lawrence S, Giles CL, Tsoi AC, Back AD (1997) Face recognition: a convolutional neural-network approach. IEEE Trans Neural Netw 8(1):98-113

35. Pannu HS, Singh D, Malhi AK (2018) Improved particle swarm optimization based adaptive neuro-fuzzy inference system for benzene detection. CLEAN-Soil, Air, Water 46(5):1700162

36. Pannu HS, Singh D, Malhi AK (2019) Multi-objective particle swarm optimization-based adaptive neuro-fuzzy inference system for benzene monitoring. Neural Comput \& Applic 31:2195-2205

37. Kaur M, Gianey HK, Singh D, Sabharwal M (2019) Multiobjective differential evolution based random forest for e-health applications. Mod Phys Lett B 33(05):1950022

38. Kaur M, Singh D, Sun K, Rawat U (2020) Color image encryption using non-dominated sorting genetic algorithm with local chaotic search based 5D chaotic map. Futur Gener Comput Syst 107:333350

39. Storn R, Price K (1995) Differential evolution-a simple and efficient heuristic for global optimization over continuous spaces (Tech. Rep.), Berkeley, CA. TR-95-012

40. Zhabitskaya E, Zhabitsky M (2012) Asynchronous differential evolution. In: Mathematical Modeling and Computational Science, pp 328-333

41. Zhang J, Sanderson AC (2009) JADE: adaptive differential evolution with optional external archive. IEEE Trans Evol Comput 13(5): 945-958

42. Vaishali, Sharma TK (2016) Asynchronous differential evolution with convex mutation. In: Proceedings of Fifth International Conference on Soft Computing for Problem Solving. Springer, Singapore, pp 915-928

43. Ilonen J, Kamarainen JK, Lampinen J (2003) Differential evolution training algorithm for feed-forward neural networks. Neural Process Lett 17(1):93-105

44. Storn R (1996) On the usage of differential evolution for function optimization. In: Fuzzy Information Processing Society, 1996. NAFIPS. Biennial Conference of the North American, pp 519 523. IEEE

45. Hancer E, Xue B, Zhang M (2018) Differential evolution for filter feature selection based on information theory and feature ranking. Knowl-Based Syst 140:103-119

46. Kaur M, Kumar V, Li L (2019) Color image encryption approach based on memetic differential evolution. Neural Comput \& Applic 31(11):7975-7987

Publisher's note Springer Nature remains neutral with regard to jurisdictional claims in published maps and institutional affiliations. 